\title{
Dynamic Inventory and Pricing Policy with Ordering and Price Adjustment Cost
}

\author{
Baimei Yang \\ School of Business \\ Shanghai Dianji University \\ Shanghai City, China \\ yangbm@sdju.edu.cn
}

\begin{abstract}
In this paper, we study a dynamic inventory control and pricing optimization problem in a periodic review inventory system with fixed ordering cost and price adjustment cost. At the same time, the ordering quantity is limited. We show that the optimal inventory control is partially characterized by an (s, s', p) policy in four regions, and the optimal pricing policy is dependent on the inventory level after the replenishment decision.
\end{abstract}

Keywords-optimization; ordering cost; price adjustment cost.

\section{INTRODUCTION}

In this article, we consider a dynamic inventory control and pricing optimization problem in a periodic review inventory system with fixed ordering cost and price adjustment cost. At the same time, the ordering quantity is limited.

This problem is related to the optimal control of a single product system with finite capacity and setup cost. Several studies have been conducted on this problem. For instance, Chen and Lambrecht (1996) point out the generally known result is that the optimal policy can only be partially characterized in the form of $X-Y$ bands. When the inventory level is below the first band $X$, then produce/order the capacity, and when the inventory level is over the second band $Y$, produce/order nothing. If the inventory level is between the two bands, the ordering policy is complicated and depends on the instance. In Gallego and Scheller-Wolf (2000), the structure of the policy between the bands is further refined using two numbers $s$ and $s^{\prime}$ in four possible regions. However, none of they have studied the pricing problem in the inventory control problem. Only Chao, Yang and $\mathrm{Xu}$ (2012) have studied a dynamic inventory and pricing optimization problem in a periodic review inventory system with setup cost and finite ordering capacity in each period. They show that the optimal inventory control is characterized by an $\left(s, s^{\prime}, p\right)$ policy in four regions of the starting inventory level. But in their paper, the selling price can be adjusted without any cost.

In the reality, there must be some price adjustment cost when the price is changed. In the economics literature, there are two major types of price adjustment costs identified: managerial costs and physical costs. Rotemberg (1982), Levy et al. (1997), Slade and G.R.E.Q.A.M (1998), Aguirregabiria (1999), Bergen et al. (2003), Zbaracki et al. (2004) have shown both the two styles of costs are significant in retailing and other industries. According to these empirical studies, Chen, Zhou and Chen (2011) consider a periodic-review inventory model with price adjustment costs that consist of both fixed and variable components. They develop the general model and characterize the optimal policies for two special scenarios, a model with inventory carryover and no fixed price-change costs and a model with fixed price-change costs and no inventory carryover. Although there is price adjustment cost, they do not consider the finite ordering capacity.

In this paper, we consider a random additive demand model and investigate the structure of the optimal inventory control and pricing policy in each period. We show that the optimal inventory policy is partially characterized by an (s, $s^{\prime}$, $p$ ) policy on four regions, in two of these regions the optimal policy is completely specified while in the other two, it is partially specified. More specifically, the optimal ordering quantity in the first region is the full capacity, while in the last region it is optimal to order nothing; in the two middle regions, the optimal decision is either to order to the maximum capacity, to order to at least a pre-specified level $s^{\prime}$, or to order nothing. The optimal pricing policy $p(y)$ in each period is dependent on the inventory level after the replenishment decision, $y$, which is in general not a monotone function. The key concept utilized is strong $C K$ concavity, which is an extension of $K$-concavity, and was first introduced by Gallego and Scheller-Wolf (2000). The result is similar with the one in Chao, Yang and Xu (2012).

The rest of this paper is organized as follows. In the next section we present the model. The main result and the proofs are provided in Section 3, and concluding remarks are in Section 4.

\section{MODEL}

Consider a periodic review inventory system with pricedependent random demand in each period. There are $N$ periods, with the first period being 1 , and last period $N$. The demand in period $n, D_{n}\left(p_{n}\right)$, depends on the selling price in period $n, p_{n}$. Here we consider the additive demand (Petruzzi and Dada (1999), Chen and Simchi-Levi (2004)). That is, the demand in period $n$ is

$$
D_{n}\left(p_{n}\right)=d_{n}\left(p_{n}\right)+\varepsilon_{n},
$$


where the selling price in period $n$ is $p_{n}, n=1, \cdots \cdots, N$. $\varepsilon_{n}$ is a random variable with mean zero and $d_{n}\left(p_{n}\right)$ is the average demand which is a decreasing function of $p_{n}$. The selling price $p_{n}$ for period $n$ is a decision variable, taken from interval $[\underline{p}, \bar{p}]$. Thus, as the selling price increases from $\underline{p}$ to $\bar{p}$, the average demand decreases from $d(\underline{p})$ to $d(\bar{p})$. Let $\bar{d}=d(\underline{p})$ and $\underline{d}=d(\bar{p})$.This shows that determining the selling price $p_{n}$ is equivalent to setting the average demand $d_{n}$, and we can simply optimize over the average demand $d_{n}$ between $\underline{d}$ and $\bar{d}$. Let $p_{n}=p_{n}\left(d_{n}\right)$ be the inverse function of $d_{n}\left(p_{n}\right)$, which is also decreasing function. Suppose $p_{n}\left(d_{n}\right)$ is linear on $d_{n}$. Then the expected revenue when setting the average demand to $d_{n}$ is

$$
r_{n}\left(d_{n}\right)=d_{n} \cdot p_{n}\left(d_{n}\right),
$$

which is a concave function. The ordering leadtime is zero, that is, an order placed at the beginning of a period is received at the end of the period after demand is realized.

We assume that the government gives the product a guide price $p_{0}$. The actual selling price $p_{n}$ should be no larger than $p_{0}$, which indicates $p_{n} \leq \bar{p} \leq p_{0}$. The cost of a price adjustment from the actual selling price to guide price in period $n$ is denoted by $U_{n}\left(p_{0}-p_{n}\right)$. It is commonly assumed that the variable cost is increases with the size of the price change, because the decision and internal communication costs are higher for larger price changes. Several forms of $U_{n}(\cdot)$ have been used in the economics literature, including piece wise linear functions and quadratic functions. Here we assume that $U_{n}(\cdot)$ is linear. Due to the linearity of decreasing function $p_{n}\left(d_{n}\right)$, it is easy to show $U_{n}\left(p_{0}-p_{n}\right)$ is linear and increases on $d_{n}$. For convenience, let

$$
U_{n}\left(p_{0}-p_{n}\left(d_{n}\right)\right)=U_{n}\left(d_{n}\right) .
$$

The sequence of events during a period is as follows: 1) inventory level is reviewed and replenishment order is placed, 2) replenishment order arrives, 3) a selling price is set, 4) random demand is realized, and 5) all costs are computed.

We assume the fixed ordering cost is $K$, and the variable unit ordering cost is $c$ in period $n$. There is a finite ordering capacity $C$ for each period. That is, the ordering quantity for each period cannot exceed $C$. Unsatisfied demand in a period is fully backlogged. Let $x_{n}$ be the inventory level at the beginning of period $n$ before placing an order, which can be positive or negative because we consider backlog model, and let $y_{n}$ be the inventory level after placing the order. Due to ordering capacity, we must have

$$
x_{n} \leq y_{n} \leq x_{n}+C
$$

A cost $h(x)$ is incurred at the end of period $n$ if the inventory level after demand realization is $x$, which represents inventory holding cost if $x \geq 0$ and shortage cost if $x<0$.

For convenience we let

$$
G(y)=E\left[h\left(y-\varepsilon_{n}\right)\right] .
$$

Then, the period- $n$ expected holding and shortage cost, given that the inventory level after replenishment is $y_{n}$ and the expected demand for period $n$ is $d_{n}$, is $G\left(y-d_{n}\right)$.

Denote by $v_{n}(x)$ the profit-to-go function at the beginning of time period $n$ with inventory level $x$. Let $v_{N+1}(x)=0$ for all $x$. Hence, for each $n=1, \cdots \cdots, N$, we have

$$
\begin{array}{r}
v_{n}(x)=c x+\max _{x \leq y \leq x+C} \max _{\underline{d} \leq d \leq \bar{d}}\left\{-K 1[y>x]+r_{n}(d)-c y\right. \\
\left.-U_{n}(d)-G(y-d)+\alpha E\left[v_{n+1}\left(y-d-\varepsilon_{n}\right)\right]\right\} \\
=c x+\max _{x \leq y \leq x+C}\{-K 1[y>x]-c y \\
\left.\quad+\max _{\underline{d} \leq d \leq \bar{d}}\left\{r_{n}(d)-U_{n}(d)+W_{n}(y-d)\right\}\right\},
\end{array}
$$

where

$$
W_{n}(y)=-G(y)+\alpha E\left[v_{n+1}\left(y-\varepsilon_{n}\right)\right]
$$

$1[A]$ is the indicate function, taking value 1 if statement $A$ is true and zero otherwise. $\alpha$ is the one-period discount factor, $\alpha \in[0,1]$.

The objective is to characterize the optimal ordering and pricing strategy that maximizes the total expected discounted profit over the planning horizon.

\section{ANALYSIS AND RESULTS}

Before analysis, it is necessary for us to introduce an important definition.

Definition: Given non-negative constants $C$ and $K$, we call the function $G$ strong $C K$-concave if for all $y, 0 \leq a<\infty$, $0<b<\infty, \forall z \in[0, C]$,

$$
-K+G(y+z) \leq G(y)+\frac{z}{b}\{G(y-a)-G(y-a-b)\}
$$

According to Gallego and Scheller-Wolf (2000), we can obtain some propositions about strong $C K$-concave:

Proposition: 1. If $G$ is strong $C K$-concave, it is also strong $D L$-concave for any $0 \leq D \leq C$ and $L \geq K$. 
2. If $G$ is concave, it is strong $C K$-concave for any nonnegative $C$ and $K$.

3. If $G_{1}$ is strong $C K$-concave, and $G_{2}$ is strong $C L$-concave, then for $\alpha, \beta \geq 0, \alpha G_{1}+\beta G_{2} \quad$ is strong $C(\alpha K+\beta L)$-concave.

4. If $G$ is strong $C K$-concave and $X$ is a random variable such that $E[|G(y-X)|]<\infty$, then $E[G(y-X)]$ is strong CK-concave.

Given non-negative $\mathrm{C}$ and $\mathrm{K}$ and strong $C K$-concave functions $g_{n}\left(x, d_{n}(x)\right)$ for any $n=1, \cdots \cdots, N$, let:

$S_{n}$ be the point at which the function $g_{n}\left(x, d_{n}(x)\right)$ reaches its maximum at period $n$, $n=1, \cdots \cdots, N$;

$s_{n}$ be the minimum point at which no order is required by the optimal policy at period $n, n=1, \cdots \cdots, N$;

$s_{n}^{\prime}$ be the maximum point at which it is necessary to order some units for the optimal policy at period $n$ and $s_{n}^{\prime} \leq S_{n}, n=1, \cdots \cdots, N$

Clearly $-\infty \leq s_{n} \leq s_{n}^{\prime} \leq S_{n}$.

Lemma 1. 1. $g_{n}\left(x, d_{n}(x)\right)$ is non-decreasing on $\left(-\infty, s_{n}^{\prime}\right]$ and strictly increasing on $\left(-\infty, s_{n}\right)$.

$$
g_{n}\left(x, d_{n}(x)\right) \geq \max _{x \leq y \leq x+C} g_{n}\left(y, d_{n}(y)\right)-K \quad \forall x>s_{n}^{\prime}
$$

3. If $s_{n}^{\prime}-C \leq s_{n}$,

$$
\begin{aligned}
& v_{n}(x)=g_{n}\left(x+C, d_{n}(x+C)\right)+c_{n} x-K, \quad x<s_{n}^{\prime}-C \\
& v_{n}(x)=\max _{s_{n}^{\prime} \leq y \leq x+C} g_{n}\left(y, d_{n}(y)\right)+c_{n} x-K, \quad s_{n}^{\prime}-C \leq x<s_{n} \\
& v_{n}(x)=\max \left\{g_{n}\left(x, d_{n}(x)\right)+c_{n} x,\right. \\
& \left.\max _{s_{n}^{\prime} \leq y \leq x+C} g_{n}\left(y, d_{n}(y)\right)+c_{n} x-K\right\}, \quad s_{n} \leq x \leq s_{n}^{\prime}
\end{aligned}
$$$$
v_{n}(x)=g_{n}\left(x, d_{n}(x)\right)+c_{n} x, \quad x>s_{n}^{\prime}
$$$$
\text { If } s_{n}^{\prime}-C>s_{n} \text {, }
$$$$
v_{n}(x)=g_{n}\left(x+C, d_{n}(x+C)\right)+c_{n} x-K, \quad x<s_{n}
$$$$
v_{n}(x)=\max \left\{g_{n}\left(x, d_{n}(x)\right)+c_{n} x,\right.
$$$$
\left.g_{n}\left(x+C, d_{n}(x+C)\right)+c_{n} x-K\right\}, \quad s_{n} \leq x \leq s_{n}^{\prime}-C
$$ 
It follows from the property of strong $C K$-concavity that $\alpha E\left[V_{n+1}\left(y-\varepsilon_{n}\right)\right]$ is strongly $C(\alpha K)$-concave, and by the concavity of $-G_{n}(y)$ we obtain that $W_{n}(y)$ is strongly $C(\alpha K)$-concave, hence it is also strongly $C K$-concave. By Lemma 3 we obtain that

$$
H_{n}(y)=-c y+\max _{\underline{d} \leq d \leq \bar{d}}\left\{r_{n}(d)-U_{n}(d)+W_{n}(y-d)\right\} .
$$

is strongly $C K$-concave. Define $s_{n}, s_{n}^{\prime}$ and $S_{n}$ by

$$
\begin{gathered}
s_{n}=\inf \left\{x \mid-K+\sup _{x \leq y \leq x+C} H_{n}(y) \leq H_{n}(x)\right\}, \\
s_{n}^{\prime}=\max \left\{x \mid-K+\sup _{x \leq y \leq x+C} H_{n}(y) \geq H_{n}(x)\right\}, \\
S_{n}=\inf \left\{y \in \Re \mid H(y)=\sup _{y \in \Re} H_{n}(y)\right\} .
\end{gathered}
$$

Then, clearly $s_{n} \leq s_{n}^{\prime}$, and it can be shown similar to the argument in Gallego and Scheller-Wolf (2000) that, the optimal inventory strategy is determined by $s_{n}$ and $s_{n}^{\prime}$ as described in the statement of Theorem 1 and that, by the strong $C K$-concavity of $H_{n}(y), v_{n}(x)$ given by

$$
v_{n}(x)=c x+\max _{x \leq y \leq x+C}\left\{-K 1[y>x]+H_{n}(y)\right\}
$$

preserves strong $C K$-concavity.

The optimal pricing decision is determined by the maximizer in Lemma 2. Let

$$
d_{n}^{*}(y)=\arg \max _{\underline{d} \leq d \leq \bar{d}}\left\{r_{n}(d)-U_{n}(d)+W_{n}(y-d)\right\},
$$

resulting in a state-dependent optimal average selling quantity. Recall that $p_{n}=p_{n}\left(d_{n}\right)$ is the inverse function of $d_{n}=d_{n}\left(p_{n}\right)$. The optimal pricing decision, when the replenished inventory level is $y$, is

$$
p_{n}^{*}(y)=p_{n}\left(d_{n}^{*}(y)\right) .
$$

This concludes the proof of Theorem 1 .

\section{CONCLUSION}

In this paper, we consider a dynamic inventory control and pricing optimization problem in a periodic review inventory system with fixed ordering cost and price adjustment cost. At the same time, the ordering quantity is limited. Here we assume that the guide price is larger than the actual selling price. And both the price function and price adjustment cost function are linear. In the future research, we will study the model with more complicate price adjustment cost.

\section{ACKNOWLEDGMENT}

This paper was supported in part by Project of Shanghai Municipal Education Commission (No. 09YS485), Humanities and Social Sciences Research Youth Foundation Project of Ministry of Education of China (No. 10YJC630274), the Natural Science Foundation of Shanghai, China (No. 10ZR1412300) and Shanghai Dianji University (No. 10XKJ01).

\section{REFERENCES}

[1] V. Aguirregabiria, "The dynamics of markups and inventories in retailing firms," The Review of Economic Studies, 1999, 66, pp. 275308.

[2] M. Bergen, M. Ritson, S. Dutta, D. Levy, and M. Zbaracki, "Shattering the myth of costless price changes," European Management Journal, 2003, 21, pp. 663-669.

[3] X. Chao, B. Yang, and Y. Xu, "Dynamic inventory and pricing policy in a capacited stochastic inventory system with fixed ordering cost," Operations Research Letters, 2012, 40, pp. 99-107.

[4] X. Chen, and M. Lambrecht, "X-Y band and modified (s, S) policy," Operations Research, 1996, 44, pp. 1013-1019.

[5] X. Chen, and D. Simchi-Levi, "Coordinating Inventory Control and Pricing Strategies with Random Demand and Fixed Ordering Cost: The Finite Horizon Case," Operations Research, 2004, 52, pp. 887896

[6] X. Chen, S.X. Zhou, and Y. Chen, "Integration of inventory and pricing decisions with costly price adjustments," Operations Research, 2011, 59, pp. 1144-1158.

[7] G. Gallego, and A. Scheller-Wolf, "Capacitated inventory problems with fixed order costs: Some optimal policy structure," European Journal of Operational Research, 2000, 126, pp. 603-613.

[8] D. Levy, M. Bergen, S.Dutta, and R. Venable, "The magnitude of menu costs: Direct evidence from large U.S. supermarket chains," The Quarterly Journal of Economics, 1997, 112, pp. 791-825.

[9] N.C. Petruzzi, and M. Data, "Pricing and the newsvendor model: A review with extension," Operations Research, 1999, 47, pp. 183-194.

[10] J. Rotemberg, "Sticky prices in the United States", Journal of Political Economy," 1982, 90, pp. 1187-1211.

[11] M.E. Slade, and G.R.E.Q.A.M, "Optimal pricing with costly adjustment: Evidence from retail-grocery prices," The Review of Economic Studies, 1998, 65, pp. 87-107.

[12] M.J. Zbaracki, M. Ritson, D. Levy, S. Dutta, and M. Bergen, "Managerial and customer costs of price adjustment: Direct evidence from industrial market," The Review Economics and Statistics, 2004, 86, pp. 512-533. 